\title{
Thermal Design of Astrobee Perching Arm
}

\author{
In-Won Park ${ }^{1}$, Trey Smith ${ }^{2}$ and John F. Love ${ }^{2}$
}

\begin{abstract}
This paper presents the thermal design of actuators in the perching arm of Astrobee robot that will operate inside the International Space Station (ISS) in starting 2019. Since the crew's safety is of the utmost importance on the ISS, all materials used in the Astrobee robot should meet the touch temperature requirements according to the ISS safety standards to protect crew from skin burns. The Astrobee perching arm consists of 2-DOF arm servo motors and 1DOF gripper DC motor, which are capable of overheating when stalled, particularly given the lack of gravity-driven thermal convection in the ISS zero-gee environment. Thermal properties of two types of actuators are verified by monitoring the touch temperature in worst-case operations with no thermal protection. Then, the proper thermal protection designs have been conducted and installed to guarantee the safety in all conditions.
\end{abstract}

\section{INTRODUCTION}

The Intelligent Robotics Group at NASA Ames Research Center is building a free-flying robot, Astrobee, which will be operated inside the International Space Station (ISS) in starting 2019 to perform a variety of intravehicular activities. Astrobee is expected to support autonomous operations, remote operation by ground controllers, and human-robot interaction with crew members [1]-[5]. Fig. 1 shows the flight unit of Astrobee docking on the charging station on the top of micro-gravity simulating surface.

\section{A. Perching Arm}

As a part of the Astrobee robotic system, a compliant, detachable perching arm is being developed to support long duration tasks. This arm will grasp ISS handrails to hold its position without using propulsion or navigation to minimize power consumption. It will also support Astrobee robots grasping each other to enable future research related to satellite servicing.

Fig. 2 shows the flight unit of 3-DOF Astrobee perching arm consists of payload lever, arm proximal joint, arm distal joint, and gripper, where the design of structure and avionics are presented in [6]. The payload levers support quick release/locking mechanism to the Astrobee payload volume. The crew does not require additional tool to install the arm to the Astrobee payload bay, where the magnets act as stop and holder for the levers. In addition, the lever is operable by one hand, which requires $5 \mathrm{~N}$ of force applied at the lever tip.

\footnotetext{
${ }^{1}$ Author is with SGT Inc. within the Intelligent Robotics Group, NASA Ames Research Center, Moffett Field, CA 94035, USA in.w.parkenasa.gov

${ }^{2}$ Authors are with NASA Ames Research Center, Moffett Field, CA 94035, USA trey.smithenasa.gov, john.f.loveenasa.gov
}

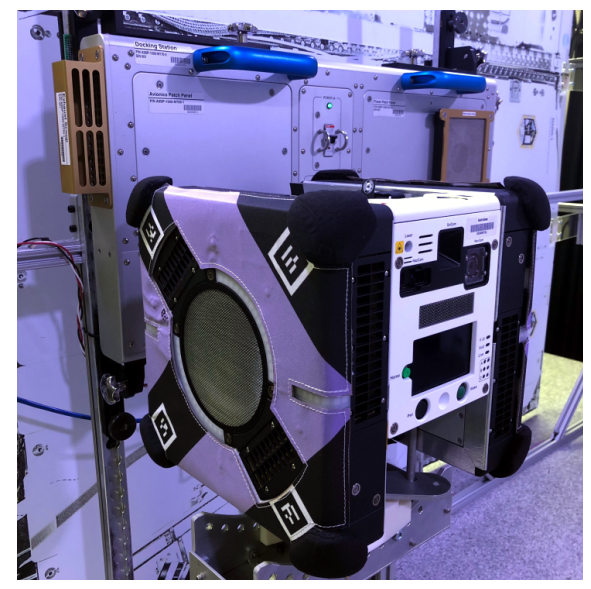

Fig. 1. Astrobee docking on the station on the top of micro-gravity simulating surface.

The 2-DOF arm joints are used to stow the gripper inside of the outer structure during flight so that it is not exposed to collision hazard while stowed. When the arm is successfully perched on the ISS handrail, it can also operate as a pan-tilt module for a camera attached on the opposite side of the robot to support remote monitoring operations [7]. The 1DOF gripper uses torsional springs for joint flexion and an actuated tendon for extension. This allows gripping force to be maintained even with the motor turned off. It also allows external forces to open the gripper by overcoming spring torques, rather than having to back-drive the motor. Furthermore, independent flexion torques at the proximal and distal joints provide passive compliance to the shape of the grasped object; the perching procedure is thus robust to positioning errors with respect to the handrail.

The 2-DOF arm servo motors and 1-DOF gripper DC motor are capable of overheating themselves if they operate near stall torque for an extended time. Thus, these actuators must ensure that either active or passive thermal controls are designed and developed to pass the ISS safety standards for touch temperature requirements. This paper presents the passive thermal control used in arm servo motors and the active thermal control used in gripper DC motor.

This paper is organized as follows. Section II describes the thermal requirement for touch temperature in the ISS. Section III presents the experimental results of arm and gripper motors tested in the worst-case operations without any thermal protection to understand the thermal properties of each actuator. Section IV explains the proposed thermal control strategy of each motor and concluding remarks follow in Section V. 


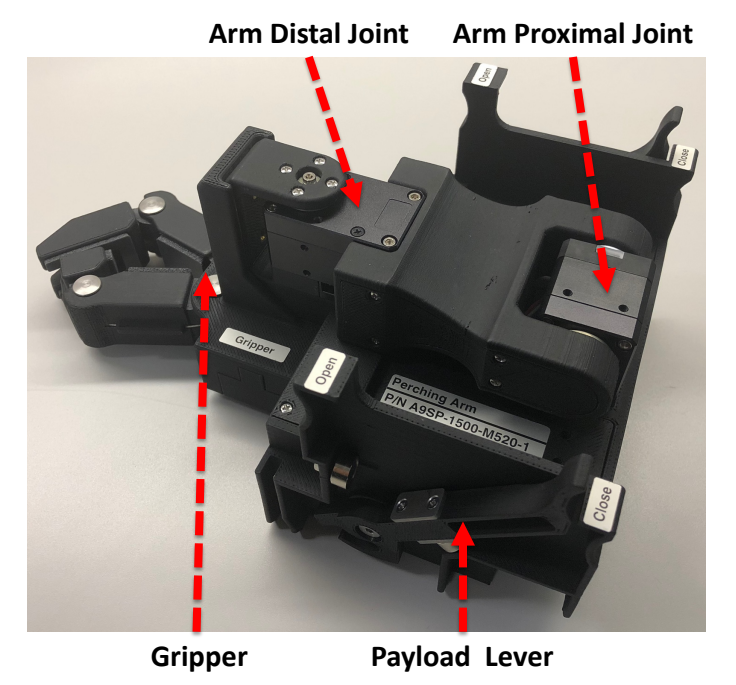

Fig. 2. Flight unit of 3-DOF Astrobee perching arm.

TABLE I

HOT TOUCH TEMPERATURE LIMITS FOR ASTROBEE MATERIALS

\begin{tabular}{c||c|c|c}
\hline \hline Material & $\begin{array}{c}\text { Incidental } \\
\text { Second }\left[{ }^{\circ} \mathrm{C}\right]\end{array}$ & $\begin{array}{c}\text { Incidental } \\
10 \text { Seconds }\left[{ }^{\circ} \mathrm{C}\right]\end{array}$ & $\begin{array}{c}\text { Infinite } \\
\text { Contact }\left[{ }^{\circ} \mathrm{C}\right]\end{array}$ \\
\hline Steel & 73 & 52 & 46 \\
Aluminum & 75 & 51 & 47 \\
Glass & 96 & 64 & 57 \\
Ultem & 122 & 76 & 68 \\
\hline \hline
\end{tabular}

\section{Thermal REQUiREMENT}

The perching arm must comply with ISS touch temperature safety requirements to protect astronauts. The human pain threshold is approximately $44^{\circ} \mathrm{C}$ at the epidermis/dermis interface. At that temperature, significant epidermal damage happens after about six hours; damage occurs much more rapidly as the temperature rises. NASA's safety approach, however, is to limit touch temperature so as to avoid reaching the pain threshold at all.

The maximum safe touch temperature to stay below the pain threshold depends on both the material properties of the touched surface and the assumed skin contact time. [8] specifies an empirical formula that applies to homogeneous materials. Table I gives sample values for materials used in the Astrobee robot. One second skin contact is a typical assumption for incidental contact brushing against items that are not meant to be handled by astronauts. Longer contact time is prevented by the human reflex to move away from a hot object.

Any component that is capable of overheating itself beyond the touch temperature limit shall have either active or passive thermal controls. If active thermal control is used, it needs to be single fault tolerant (two independent controls $A$ and $B$ ). To verify single fault tolerance, we can disable thermal control $B$ and execute a worst-case operation to maximize the exterior temperature, comparing to the touch temperature limit and verifying $A$. Then the test should be repeated with $A$ disabled in order to verify $B$.

Arm motors for proximal and distal joints and gripper
TABLE II

PARAMETER SETUP OF ARM MOTORS AND CURRENT CONSUMPTION IN STALL CONDITION

\begin{tabular}{c||c|c|c}
\hline \hline $\begin{array}{c}\text { Test } \\
\text { Condition }\end{array}$ & $\begin{array}{c}\text { PWM Limit } \\
{[\%]}\end{array}$ & $\begin{array}{c}\text { Temperature } \\
\text { Limit }\left[{ }^{\circ} \mathrm{C}\right]\end{array}$ & $\begin{array}{c}\text { Current } \\
\text { Consumption [A] }\end{array}$ \\
\hline$(1)$ & 100 & 80 & 1.58 \\
$(2)$ & 80 & 80 & 1.12 \\
$(3)$ & 70 & 80 & 0.90 \\
$(4)$ & 50 & 80 & 0.64 \\
$(5)$ & 50 & 80 & 0.65 \\
$(6)$ & 50 & 100 & 0.60 \\
\hline \hline
\end{tabular}

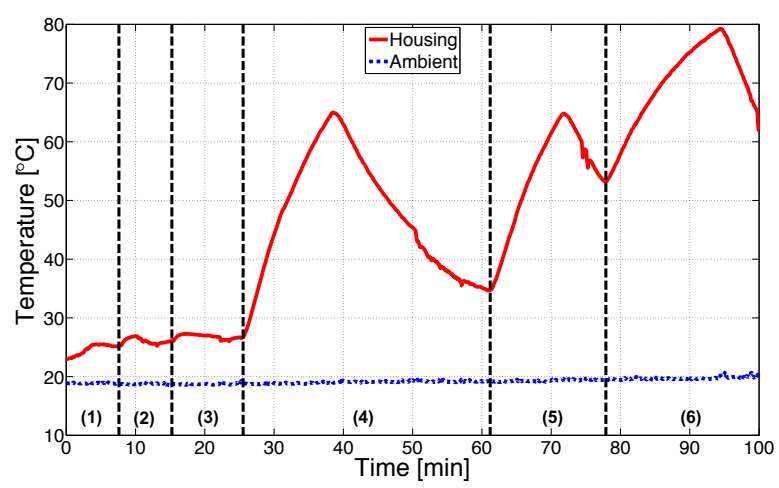

Fig. 3. Surface temperature of arm motor in stall condition.

motor are the potential heat sources as shown in Fig. 2 . The arm servo motor (Dynamixel XH430-W210) has an aluminum case, which acts as a good heat sink, but it is exposed to the crew and is cooled primarily by radiation to the ISS environment. An overheating event may occur when the arm motor goes into a prolonged stall, when the PWM limit is set incorrectly or not handled properly, or when the maximum internal temperature limit fails. Note that the last two cases could be triggered by a bug in the proprietary COTS firmware. Going by ISS safety rules, our overall design cannot rely on proper functioning of the firmware to control a safety hazard unless the firmware is certified to safety-critical software standards, which would be infeasible in this case. Therefore we try to plan for worst-case software behavior.

The gripper DC motor (Pololu Micro Gearmotor) is enclosed in Ultem and not exposed to the crew, but the touch temperature on the enclosure should be monitored while actuating the motor near the stall condition.

\section{Results Without Thermal Protection}

This section describes the experimental results of arm motor and gripper motor to understand the thermal properties. Both motors are tested in the worst-case operations without any thermal protection to maximize the metal housing temperature.

\section{A. Arm Motor - Dynamixel XH430}

In the test setup, the thermocouples are placed on the top of external aluminum surface and the arm link to measure the touch temperature, and one on the table to measure ambient 
temperature. At the end of arm link, $60 \mathrm{~g}$ of dummy mass has been added to create a resistance torque and to simulate the inertia of the Astrobee during a panning motion aboard the ISS. In addition to the thermocouples, the perching arm controller board has monitored both the internal motor temperature reported by the COTS firmware and current consumption by the current sensor.

In the normal operating condition, the internal and external temperature are reached to $40^{\circ} \mathrm{C}$ and $31^{\circ} \mathrm{C}$, respectively, and $0.19 \mathrm{Ah}$ of current is consumed after 47 minutes when the temperature is reached near equilibrium while the motor is continuously moving. If one assumes the maximum ISS internal temperature is $30^{\circ} \mathrm{C}$, the temperature can increase up to $17^{\circ} \mathrm{C}$ because the touch temperature of aluminum for infinite contact is $47^{\circ} \mathrm{C}$ as shown in Table. I. The ambient temperature at the start of the test is $19^{\circ} \mathrm{C}$ meaning that as long as the temperature stays below $36^{\circ} \mathrm{C}$, the touch temperature limit would not be reached. Since the external temperature measured from thermocouple was $31^{\circ} \mathrm{C}$, the arm motor has been passed the normal operating conditions. During the test, heat has been transferred to the arm link, but the highest temperature was the aluminum surface of arm motor.

Table. II indicates the parameter setups for various test conditions and the corresponding current consumption in the stall condition. Fig. 3 shows the temperature of external motor surface measured from the thermocouple. The arm joint is clamped to the table and the initial test is ran with the internal temperature limit set to default, which is $80^{\circ} \mathrm{C}$, and the maximum PWM limit in the COTS firmware. The current reading has increased rapidly to a max of about 1.58 $\mathrm{A}$, but fluctuates about $1.5 \pm 0.08 \mathrm{~A}$, which is above the stall current 1.3 A. After 10 seconds, the current reading has returned to a low value of about 0.09 A showing that the arm motor has stopped. As shown in Table. II and Fig. 3, the COTS firmware automatically disables the motor power until the value of PWM limit has been changed to 50\%.

When value of PWM limit has been set to $50 \%$, the external aluminum surface temperature kept increasing until the internal temperature limit of COTS firmware has reached. When the internal temperature limit has set to $80^{\circ} \mathrm{C}$, the difference between the internal temperature provided by the COTS firmware and the measured external temperature was $15.0^{\circ} \mathrm{C}$ and $15.2^{\circ} \mathrm{C}$ in test conditions 4 and 5 , respectively. However, the difference has been increased to $20.7^{\circ} \mathrm{C}$ when the temperature limit has been increased to $100^{\circ} \mathrm{C}$. Since the resulting temperature values are higher than the touch temperature limits for aluminum in Table I, it would be necessary to design the thermal control that does not rely on the firmware.

\section{B. Gripper Motor - Pololu Micro Gearmotor}

Fig. 4 shows the current consumption of gripper motor in the normal operating condition. It consumes the peak maximum current of $0.62 \mathrm{~A}$ and $0.50 \mathrm{~A}$ each time for actuating the tendon to open the gripper by overcoming torsional spring forces. It would require about 0.2 A to hold

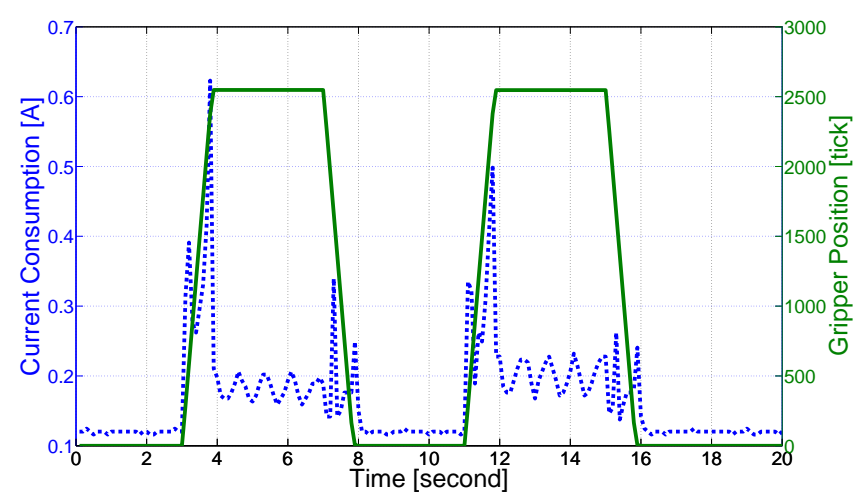

Fig. 4. Current consumption of gripper motor in normal condition.

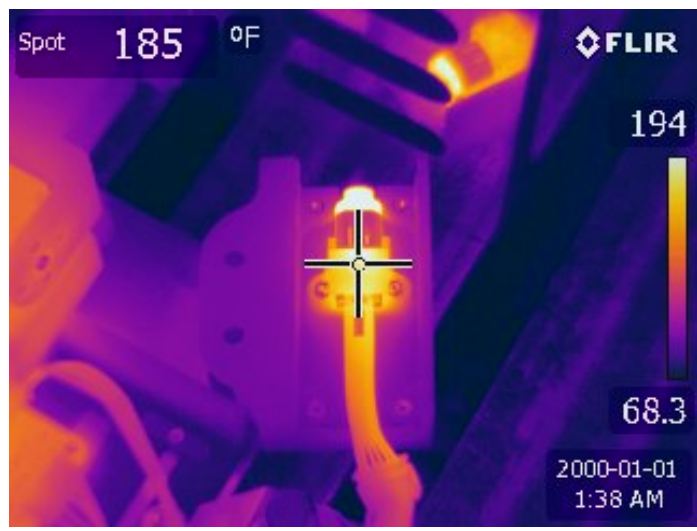

Fig. 5. Surface temperature of gripper motor in over-current condition.

the gripper open, which is well below the stall current of 0.8 A.

Fig. 5 shows the extreme temperature increase on the gripper motor surface when the over-current above the stall current is applied to the motor. In this condition, the motor has ceased to function and the surface temperature has been increased above the touch temperature limit. Even though the gripper motor is enclosed in Ultem and not exposed to the crew, the motor would require the thermal control to prevent overcurrent indicative of locked up, stalled, or jammed condition. Accidentally burning out the gripper motor has been a good lesson learned that provided extra motivation for the over-current design.

\section{Thermal Design}

This section introduces the thermal design of arm motor and gripper motor that guarantees safety in all conditions.

\section{A. Arm Motor - Bimetallic Thermostat Selection}

The perching arm motors for panning and tilting joints are exposed to the crew and are cooled primarily by radiation to the ISS environment, but also conduction through Ultem 9085 links back to the Astrobee structure. The motors do not exceed the touch temperature limit in normal operations, but in some cases, the temperature on the metal motor housing has gone up above this limit in stall conditions as shown in Fig. 3. Both arm motors provide the load information on 
force exerted by arm and stop applying the torque if resistance detected. In addition, the internal temperature sensors cut off the power if it exceeds the preset temperature limit. However, both these cutoffs are implemented in firmware, so we cannot rely on them as safety controls given that the COTS firmware has not been certified as safety-critical software.

In order to guarantee the passive thermal safety in all conditions, a bimetallic thermostat is placed on the metal motor housing, which monitors the surface temperature and cuts off the power if it exceeds the preset temperature. When the bimetallic thermostat is heated, the internal stress causes the bimetallic disc to reverse its curvature with a snap-action to open the electrical contacts of motor power. When it is below the preset temperature, the bimetallic disc relieves the internal stress and returns its curvature to normal operation condition. The specifications of bimetallic thermostat are given in terms of operating temperature $\left(T_{\text {open }}\right)$, differential $(D)$, and error tolerance $(E)$. Based on these specifications, the expected two behaviors of thermostat are as follows:

- When closed, the thermostat opens at nominal temperature, $T_{\text {open }}$

- When opened, the thermostat closes at nominal temperature, $T_{\text {open }}-D$

where both of these temperature threshold values have error bars of $\pm E$.

The following extra parameters are considered for analysis:

- Let $T_{\text {touch }}$ be the temperature of the hottest point on the metal motor housing that is exposed to inadvertent crew touch.

- Let $T_{\text {interface }}$ be the temperature of the thermostat contact point on the metal motor housing.

- Let $T_{\text {switch }}$ be the temperature of the thermostat.

- Let $\Delta_{1}=T_{\text {touch }}-T_{\text {interface. The size of arm motor }}$ is small and its rate of heating is limited. Thus, the entire metal motor housing should have fairly consistent temperature equalized through thermal conduction. The thermostat contact point may actually be hotter than any point exposed to crew contact, because exposure to the cabin provides a cooling effect. We have conservatively bounded, $\Delta_{1}= \pm 3^{\circ} \mathrm{C}$

- Let $\Delta_{2}=T_{\text {interface }}-T_{\text {switch. }}$. The thermostat has low thermal inertia and is in direct metal-to-metal contact with the motor housing. Since the motor is the heat source, the thermostat should be cooler than the motor housing. We have conservatively assumed, $0<\Delta_{2}<$ $3^{\circ} \mathrm{C}$.

- Let $T_{\text {hazard }}$ be the threshold value for a touch temperature hazard on the metal motor housing exposed to crew. Per the ISS safety requirement shown in Table I, we are using the threshold for 1 second inadvertent touch on aluminum, $T_{\text {hazard }}=75^{\circ} \mathrm{C}$.

- Let $T_{\text {ambient }}$ be the maximum ambient temperature in the ISS cabin. We have assumed, $T_{\text {ambient }}=30^{\circ} \mathrm{C}$.

- Let $T_{\text {nominal }}$ be the hottest $T_{\text {touch }}$ can get during the nominal operations. The worst-case observed $\Delta T$ was approximately $17^{\circ} \mathrm{C}$. This $\Delta T$ has been added to the worst-case ambient temperature to get $T_{\text {nominal }}=47^{\circ} \mathrm{C}$.

The key safety requirement is that the thermostat shall open when there is a touch temperature hazard, which is defined as follows:

$$
T_{\text {open }}+E+\Delta_{1}+\Delta_{2}<T_{\text {hazard }}
$$

where $T_{\text {open }}+E$ represents the highest thermostat temperature when thermostat open. Using the maximum values in the possible ranges for $\Delta_{1}$ and $\Delta_{2},(1)$ is reduced as follows:

$$
T_{\text {open }}<69^{\circ} \mathrm{C}-E
$$

In order to enable the nominal operation of perching arm, the thermostat shall not open during nominal operations, which is defined as follows:

$$
T_{\text {open }}-E>T_{\text {nominal }}-\Delta_{1}-\Delta_{2}
$$

where $T_{\text {open }}-E$ represents the lowest thermostat temperature when thermostat is opened and $T_{\text {nominal }}$ represents the highest temperature during nominal operations. Using the minimum values in the possible ranges for $\Delta_{1}$ and $\Delta_{2}$, (3) is reduced as follows:

$$
T_{\text {open }}>50^{\circ} \mathrm{C}+E
$$

Ideally, in the unlikely event that the thermostat ever trips, the thermostat shall close after an extended period of nonoperation to restore the functionality of perching arm, which is defined as follows:

$$
T_{\text {open }}-D-E>T_{\text {ambient }}
$$

where $T_{\text {open }}-D-E$ represents the lowest thermostat temperature when thermostat is closed and $T_{\text {ambient }}$ represents the highest thermostat temperature after extended non-operation. Using the maximum ambient temperature in the ISS cabin, (5) is reduced as follows:

$$
T_{\text {open }}>30^{\circ} \mathrm{C}+D+E
$$

Having plenty of design margin is more important with regard to the top end of the range, because if there is any issue with the bottom end of the range, there is an option to mitigate it with the operations rule on the duty cycle of arm operations. This leads to the following rules for selecting the optimal thermostat:

- Prefer the differential $D$ and error tolerance $E$ to be as small as possible.

- Choose the lowest $T_{\text {open }}$ that still satisfies both constraints (4) and (6).

- Verify constraint (2) is satisfied.

Based on the datasheet, Klixon M1130110122 has been selected and it has been placed on the bottom surface of each arm motor as shown in Fig. 6. This thermostat has $T_{\text {open }}=130^{\circ} \mathrm{F}\left(\approx 54.4^{\circ} \mathrm{C}\right), T_{\text {open }}-D=110^{\circ} \mathrm{F}\left(\approx 43.3^{\circ} \mathrm{C}\right)$ with the tolerance of $\pm E=2.8^{\circ} \mathrm{C}$. During installation, thermal conductive adhesive (3M TC-2810) is applied on the face of thermostat that contacts the motor to fill the 


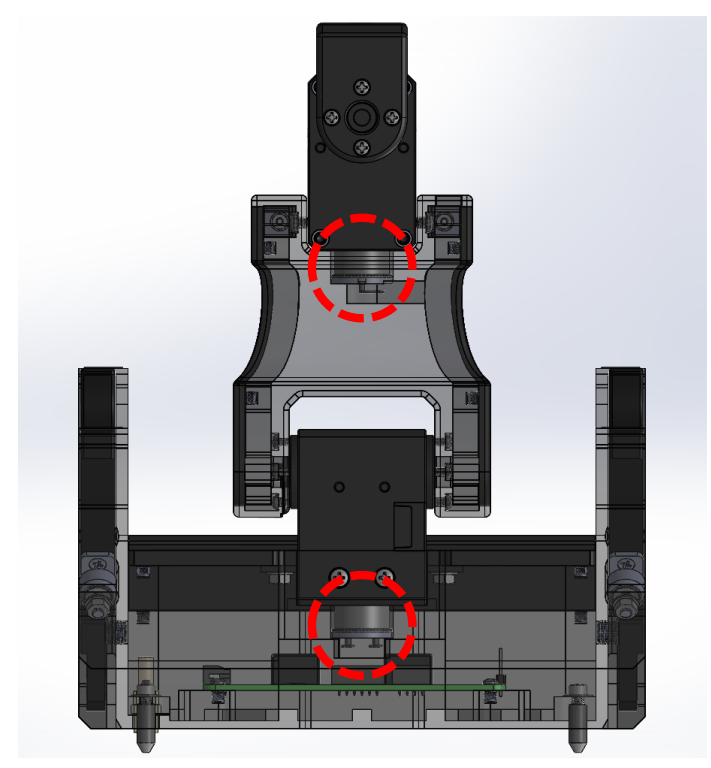

(a) Location of Bimetallic Thermostat

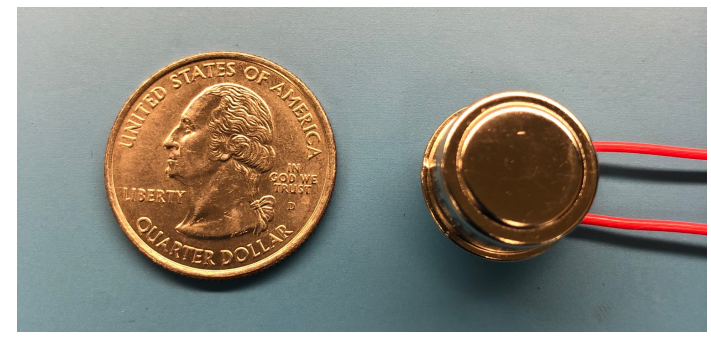

(b) Snapshot of Bimetallic Thermostat

Fig. 6. Passive thermal design on arm motors.

possible gap. Since the safety of this design does not depend on the over-temperature cutoff feature built into the COTS arm motor firmware, we decided to set that firmware cutoff threshold very high, so it has no chance of disrupting nominal operations.

\section{B. Gripper Motor - Current Limiter Circuit}

Gripper DC motor is encased and air gap insulated in Ultem 9085 , not touchable by the crew. However, as shown in Fig. 5, the metal motor housing reaches to $85^{\circ} \mathrm{C}\left(\approx 185^{\circ} \mathrm{F}\right)$ in the over-current condition without any thermal protection. The controller board is capable of monitoring the current that is being applied and stop applying the torque if the load is higher than preset threshold. However, as mentioned in Section II, any firmware involved in thermal control becomes safety critical.

For the gripper motor, an active thermal control approach is used that requires to enforce touch temperature limits are single fault tolerant. The first control approach is verified by monitoring the touch temperature while actuating the gripper motor near the maximum current level in the normal operations. As shown in Fig. 4, the peak maximum current was below the stall current. The second control approach is verified by obstructing motion in a worst-case stall condition. In order to prevent over-current due to locked up, stalled, or jammed condition, we use the over-current cutoff circuit shown in Fig. 7.

The objective of the over-current cutoff is to persistently disable the motor when an over-current fault is detected, but allow the micro-controller (dsPIC33E) to explicitly clear the fault later. This fault memory behavior is implemented using an SR flip flop (74LVC2G02). When both S and R inputs are low, the EN (enable) output holds whatever value it was previously set to. When the $\mathrm{R}$ input is high (over-current), the EN output is reset to low, disabling the motor. When the $\mathrm{S}$ input is high (clear fault), the EN output is set to high, re-enabling the motor. If both $\mathrm{S}$ and $\mathrm{R}$ are high at the same time, the flip flop behavior is indeterminate, so we avoid this condition. In practice, the micro-controller firmware sends "clear fault" on the S input only briefly during its boot sequence, so the higher-level software can effectively clear a fault by power cycling the arm.

To detect over-current, a comparator (MAX921) compares the current feedback of the motor driver (MC33926) to the reference voltage, which is set to $90 \%$ of the stall current. To clean up the noisy comparator output, we use a multi-vibrator (SN74LVC1G123) that sends a short rising edge pulse to the SR flip flop.

\section{CONCLUSIONS}

A perching arm payload was designed for the ISS freeflying robot project called Astrobee. The perching arm enables the free-flyer to grab onto the ISS hand rails and allows for pan and tilt camera positioning. Pre-compliance touch temperature testing of the perching arm revealed the possibility of the arm servo motor and the gripper DC motor exceeding the allowable material temperature during stalled conditions. Hardware, not software, solutions were designed to handle the potential stalled conditions so that original non-flight critical software architecture was maintained. A bimetallic thermostat was added to the arm servo motor in the event of firmware malfunction resulting in over-current and heating during stalled conditions. A current limiting circuit was designed for the gripper DC motor to prevent overcurrent and associated heating during stalled conditions. With the proposed thermal designs, there is no way for the exposed surface of Astrobee perching arm to exceed above the touch temperature limits, without relying on firmware controls.

\section{ACKNOWLEDGMENT}

We would like to thank the ISS Payloads Office, the JSC Flight Operations Directorate, ISS Avionics and Software, the Advanced Exploration Systems program, the ISS SPHERES team, and the Astrobee team for their collaboration.

This research was supported by the NASA Game Changing Development Program (NASA Space Technology Mission Directorate) and the ISS SPHERES Facility (NASA Human Exploration and Operations Mission Directorate). 


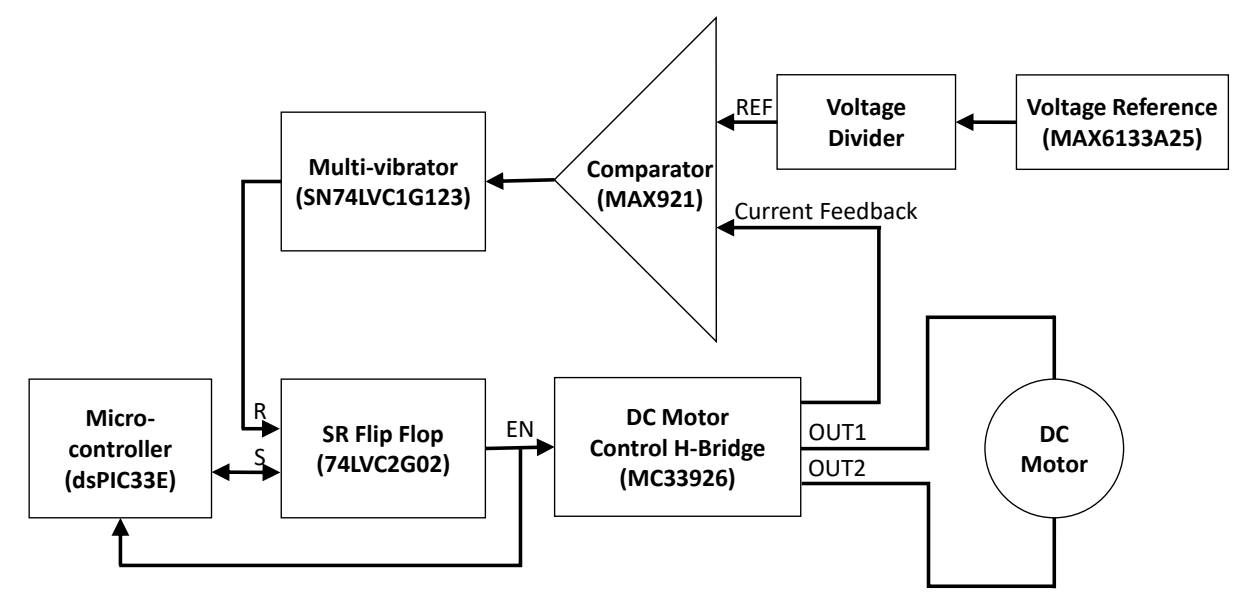

Fig. 7. Active thermal design of gripper motor using the current limiter circuit.

\section{REFERENCES}

[1] M. Bualat, J.Barlow, T. Fong, C. Provencher, T. Smith and A. Zuniga "Astrobee: Developing a free-flying robot for the international space station," in Proc. AIAA Space 2015, Aug. 2015, pp. 1-10.

[2] J. Yoo, I.-W. Park, V. To, J. Lum and T. Smith, "Avionics and perching systems of free-flying robots for the international space station," in Proc. IEEE Int. Symp. Sys. Eng., Sept. 2015.

[3] T. Smith, J. Barlow, M. Bualat, T. Fong, C. Provencher, H. Sanchez, E. Smith and the Astrobee Team, "Astrobee: A new platform for freeflying robotics on the international space station," in Proc. Int. Symp. Artificial Int. Robot. Autom. Space, Jun. 2016.

[4] B. Coltin, J. Fusco, Z. Moratto, O. Alexandrov and R. Nakamura,
"Localization from visual landmarks on a free-flying robot," in Proc. IEEE/RSJ Int. Conf. Intell. Robot. Syst., Oct. 2016, pp. 4377-4382.

[5] Evan Ackerman, IEEE Spectrum, How NASA's Astrobee robot is bringing useful autonomy to the ISS, Available: http://spectrum.ieee.org/automaton/robotics/space-robots/how-nasaastrobee-robot-is-bringing-useful-autonomy-to-the-iss

[6] I.-W. Park, T. Smith, S. W. Wong, P. Piacenza and M. Ciocarlie, in Proc. IEEE Int. Conf. Adv. Int. Mech., Jul. 2017, pp. 1135-1141.

[7] D.-H. Lee, B. Coltin, T. Morse, I.-W. Park, L. Fluckiger and T. Smith, "Handrail detection and pose estimation for a free-flying robot," Int. J. Adv. Robot. Sys., vol. 15, no. 1, pp. 1-12, 2018.

[8] E. Ungar and K. Stroud, "A new approach to defining human touch temperature standards," in Proc. Int. Conf. Env. Syst., Jul. 2010, pp. $11-15$. 